\title{
Evaluación de la gestión pública: conceptos y aplicaciones en el caso latinoamericano*
}

críticos de la reforma del sector público. Así lo sugieren los esfuerzos realizados bajo el manto de la Nueva Gestión Pública o Neo-Gerencialismo (Rhodes, 1991; Kettle, 1997; Bresser Pereira, 1997) en Nueva Zelanda, Gran Bretaña y más recientemente los Estados Unidos con el National Performance Review y el Government Performance Results Act de 1993 (Lynn, 1998).

En los países latinoamericanos, la naturaleza de estos esfuerzos responde a las particularidades históricas de la región. Por ejemplo, el problema de la calidad de la gestión pública y su capacidad para implementar políticas y movilizar recursos fueron factores críticos dentro de las reformas de segunda generación posteriores a los ajustes macroeconómicos de los años 80 (Naim, 1995). Más aún, la evidencia sugiere que el éxito de los procesos de modernización del Estado en la región depende de la creciente capacidad de sus instituciones públicas, y esta capacidad, a su vez, es un factor clave para fortalecer y profundizar la democracia y el desarrollo humano y sostenible (Prats, 1997).

La aplicación de una perspectiva sistémica de evaluación representa una agenda urgente dentro del esfuerzo por avanzar en el proceso de modernización del Estado en la región. Sin duda, existen en la actualidad importantes esfuerzos para crear indicadores de gestión en organizaciones públicas en toda América Latina. Sin embargo, son pocos los casos en los cuales se puede hablar de la utilización sistemática de estos indicadores para evaluar la gestión de organizaciones, programas y políticas públicas.

Sonia Ospina Bozzi es directora del Programa de Doctorado y profesora en la Escuela de Post Grado de Administración Pública en la Universidad de Nueva York.

Contato: sonia.ospina@ nyu.edu 
Ello presupone instrumentos de evaluación con capacidad para cubrir los

niveles micro y meso de la gestión, enmarcados dentro del contexto más amplio de la acción estatal y de sus interacciones con otros actores de la sociedad civil. Entre los esfuerzos en la región que apuntan a desarrollar una perspectiva sistémica se destacan los casos de Chile, Colombia y Costa Rica (CEPAL, 1998).

En este trabajo utilizaré los dos casos sudamericanos como punto de referencia para reflexionar sobre la naturaleza de la evaluación de la gestión pública. El objetivo es explorar la tesis de que esta práctica representa más que una simple técnica administrativa, puesto que cumple una función política como herramienta de modernización del Estado. Para abordar este objetivo, desarrollo un análisis comparativo entre los casos de Chile y Colombia a partir de tres preguntas sobre la evaluación de la gestión pública, que permitirán analizar tanto los elementos conceptuales como la naturaleza de las prácticas en contextos particulares. Son ellas:

- ¿Para qué evaluar?

- ¿Qué evaluar y a qué nivel hacerlo?

- ¿Qué hacer con la información?

Respuestas a la primera pregunta permiten explorar las múltiples funciones de la evaluación de la gestión pública, y por tanto, la lógica para abordar esta tarea en el contexto latinoamericano; la segunda pregunta explora aspectos relacionados con el objeto y los niveles de la evaluación, y por tanto, de su alcance; y la tercera se concentra en la relación entre la información producida por la evaluación y el mejoramiento de la gestión dentro de una agenda más amplia de desarrollo institucional.

A partir de una reflexión sobre estas tres preguntas y con la ayuda de observaciones sobre casos específicos, propongo el siguiente argumento: la evaluación de la gestión pública pasa de ser un ejercicio técnico a ser una herramienta política en la medida que abarque los tres niveles de la acción social, el macro, meso y micro, y en la medida que genere capacidad gerencial para establecer conexiones entre estos niveles. Esto garantiza que quienes toman decisiones pertinentes al nivel macro de la acción estatal puedan utilizar información de los niveles inferiores, y viceversa, creándose así una mayor coherencia en los distintos niveles de la administración pública y entre la gestión y las políticas públicas.

Las reflexiones y ejemplos presentados aquí tienen su origen en dos trabajos exploratorios de investigación empírica realizados por la autora durante los últimos años. Mientras que el estudio del caso colombiano se encuentra en estado más avanzado, el caso chileno se encuentra en la etapa preliminar de desarrollo. Por esta razón, este trabajo ofrece simplemente algunas ideas para estimular la discusión y demostrar así la urgencia de desarrollar una agenda comprensiva de investigación sobre las valiosas experiencias que existen en la región. En la medida de lo 
posible, el trabajo busca también utilizar el método comparativo para empezar a formular proposiciones tentativas sobre los avances en estos dos países en el área de la avaluación de la gestión pública, y la contribución de estos esfuerzos para comprender mejor la naturaleza de esta tarea y su relevancia para el proceso de modernización del Estado en América Latina.

El trabajo está, entonces, dividido en cuatro secciones. Tres de ellas exploran cada una de las preguntas planteadas y se desarrollan después de una sección inicial que proporciona una breve reseña del contexto histórico y disciplinario dentro del cual se enmarca la creciente preocupación por el tema, en el contexto mundial en general y latinoamericano en particular.

\section{Un nuevo énfasis en el problema de la evaluación: el contexto histórico y disciplinario}

Aunque el tema de la evaluación de proyectos y programas públicos no es nuevo, la preocupación actual por la evaluación de la gestión pública se enmarca dentro de la agenda más amplia de la reforma del Estado y la modernización de sus instituciones. En realidad, se puede hablar de un movimiento que propone un cambio de paradigma en la administración pública, cuyas manifestaciones concretas varían considerablemente de país a país, pero también cuyas bases filosóficas y conceptuales tienen visos comunes (Cabrero M., 1995; Ospina, 1999b). La difusión de este movimiento, que se inicia en el Reino Unido, Australia y Nueva Zelanda, y luego se desarrolla con matices diferentes en los Estados Unidos, también ha influenciado las prácticas de gestión de países de otras áreas geográficas y con otros niveles de desarrollo, como Malaysia y Singapur en el Asia, y el Brasil en América Latina (Banco Mundial, 1997; Caiden y Caiden, 1997).

Em la base de las nuevas ideas se encuentra una preocupación generalizada por los retos impuestos por un entorno cada vez más turbulento frente a la necesidad imperiosa de reevaluar el papel del Estado y de mejorar la eficiencia, eficacia y calidad de los servicios públicos. En parte, el mejoramiento de la administración pública es pre-requisito para recuperar la legitimidad del Estado, en su papel, ahora más reducido pero igualmente crítico, como co-actor en la resolución de los problemas colectivos en la sociedad de cambio de siglo.

Como resultado de esta macro-preocupación, aparece una micropreocupación por el desempeño de los empleados y las organizaciones públicas. Entre las características comunes del nuevo tipo de gestión deseado para administrarlas, podemos mencionar: 
- un mayor énfasis en los resultados producidos por las organizaciones más que en los insumos o en el proceso para conseguirlos (y por tanto, un mayor énfasis en la efectividad e impactos de la gestión);

- una mayor atención al punto de vista de los clientes/consumidores de los servicios producidos más que a los empleados o productores del servicio (y por tanto, un mayor énfasis en la calidad de los servicios).

A su vez, el nuevo paradigma genera las siguientes tendencias en la práctica de la gestión:

- $\quad$ necesidad de clarificar y definir las expectativas en relación con el desempeño de las organizaciones, y las políticas y programas que sus gerentes administran;

- necesidad de dar más flexibilidad y autonomía al gerente público, pero también pedir más responsabilidad y un claro rendimiento de cuentas frente al desempeño de su organización;

- $\quad$ reducción del tamaño del Estado y su participación directa en la producción de servicios, reemplazando ésta por mecanismos tales como la privatización y los contratos con otros proveedores;

- tratamiento de las agencias públicas como repositarias de "contratos" o "acuerdos" con los ministerios u otras jurisdicciones bajo cuya jurisdicción funcionan.

Dadas estas tendencias, se explica la motivación por desarrollar una gestión orientada a resultados y la consecuente preocupación por la evaluación de la gestión pública. En el caso de América Latina, el Consejo Científico del CLAD, en su documento "Una Nueva Gestión Pública para América Latina" (1998), identifica como una de las características y objetivos de la reforma gerencial su orientación hacia el control de los resultados. Partiendo de la precariedad del desarrollo de este aspecto de la gestión en la región, el Comité Científico del CLAD plantea que "los Estados latinoamericanos precisarán montar sólidos sistemas de evaluación del desempeño en los próximos años, en el caso de que quieran en realidad transformar las actuales estructuras de la administración pública” (p. 17).

El contexto descrito arriba proporciona un marco para empezar a entender la lógica de la evaluación, concebida ésta como una práctica administrativa deseable en la gestión pública contemporánea. Sin embargo, cabe preguntarse si su aplicación en América Latina representa un simple caso de transferencia técnica o si, más bien, las particularidades del proceso de transformación en la región hacen de la evaluación de la gestión pública una herramienta útil para el proceso de fortalecimiento de la democracia.

La descentralización fiscal, política y administrativa; la democratización y las reformas electorales; y la modernización del Estado y su administración pública representan procesos de la reforma que cambian las reglas del juego en la región, y afectan las relaciones horizontales (Estado-sociedad civil) y verticales (Estado nacional, regional y local) entre 
los actores participantes en la búsqueda de soluciones a los problemas públicos. Esta dinámica a su vez está transformando radicalmente el tipo de soluciones articuladas y las prácticas de gestión para implementarlas (Campbell,1997; Ospina, 1999a). La lógica de conceptualización e implementación de los mandatos para crear los sistemas de evaluación que aparecen dentro de este contexto está directamente enmarcada en estos cambios y responde a los retos que ellos suponen. ${ }^{1}$

Por ejemplo, la transferencia de responsabilidades fiscales y administrativas hacia los niveles regionales y locales genera la necesidad de clarificar las reglas del juego frente a la toma de decisiones públicas y sus consecuencias, así como los mecanismos para rendir cuentas sobre ellas (accountability). Esta clarificación es necesaria dentro de cada nivel (nacional, regional y local); entre cada nivel y los otros (relaciones gubernamentales); y entre los representantes del Estado y la sociedad civil en cada nivel. Igualmente, con el proceso de democratización se articulan nuevas demandas por el manejo transparente de los procesos y por más información pública acerca de los productos y el impacto de la gestión.

Finalmente, los varios procesos de modernización del Estado están intimamente interrelacionados, como lo ilustra el último informe de la CEPAL (1998) sobre la reforma fiscal, cuando sugiere que: “...ni las políticas mejor formuladas y diseñadas — en materia tributaria o del gasto público - tienen mayores posibilidades de alcanzar resultados satisfactorios si el modelo de administración pública que las pone en marcha no está sustentado en un sistema de incentivos, control y evaluación adecuado para los fines propuestos" (p. 262). Esta es la lógica dentro de la cual se justifica la preocupación por mejorar la gestión pública en general y por garantizar su efectiva evaluación en particular.

Los casos de Colombia y Chile ilustran la variedad de respuestas a estos desafíos. Efectivamente, ellos representan dos estrategias diferentes para asumir el mismo reto. En Colombia, esta política se enmarca dentro de un mandato constitucional a partir de la reforma de 1991, mientras que en Chile, ella surge como parte del esfuerzo del ejecutivo por modernizar la gestión, y solamente más adelante se codifican legalmente algunas de las iniciativas del proceso. Los tiempos y entidades responsables también varían. En Colombia se crea en 1993 una oficina especial en el Departamento Nacional de Planeación, entidad encargada hasta entonces de la evaluación de proyectos realizados con fondos públicos. En Chile, el esfuerzo es asumido en 1994 por el Comité Interministerial de Modernización de la Gestión Pública (CIMGP) del Ministerio de la Secretaría General de la Presidencia (SEGPRES), con el apoyo técnico de la Dirección de Presupuestos del Ministerio de Hacienda, entidad que ya había empezado a trabajar el problema de la evaluación desde antes. 
Los dos países han desarrollado un conjunto de actividades que demuestran su compromiso con la agenda de promover una gestión pública orientada a resultados y con su prioridad para montar sistemas de medición de estos resultados. Es posible sugerir, sin embargo, que la localización institucional de cada esfuerzo, por si misma, determinó la dirección y el abordaje de muchos de los detalles de su diseño e implementación. Como consecuencia, el producto de estos compromisos es bastante idiosincrático.

En el caso colombiano, desde un principio el énfasis se pone en la creación de un Sistema Nacional de Evaluación de la Gestión - SINERGIA - que contribuya al proceso de modernización del Estado. Ello guía las decisiones, que son tomadas desde una organización de carácter técnico más que político. En el caso chileno, el énfasis se pone en la creación de un programa sistemático de modernización de la gestión pública, cuyas múltiples iniciativas incluyen acuerdos institucionales que requieren un fuerte componente de evaluación del desempeño. Ello guía las decisiones tomadas conjuntamente por dos organizaciones, una de carácter más político y la otra con orientación más técnica.

Finalmente, en cuanto al diseño e implementación, para 1998 representantes del gobierno de Chile hablan de la existencia de un sistema en operación, lo cual significa una fase de conceptualización y diseño muy corta; mientras que para la misma fecha, existe en Colombia un sistema nacional cuya conceptualización es altamente sofisticada, pero cuya operatividad no es tan clara y su implementación se encuentra casi paralizada, además de que su relevancia ha sido cuestionada con el cambio de gobierno.

Aunque este trabajo no pretende hacer una evaluación de los dos casos, el contexto presentado aquí sugiere que su conocimiento puede ayudar a comprender mejor la naturaleza de la evaluación, no sólo como práctica administrativa y herramienta de modernización, sino también como instrumento político de consolidación de la institucionalidad. Este trabajo explora más detenidamente algunos de los desafíos y dimensiones que tal agenda ha impuesto para los países de la región. En este contexto, respuestas a las preguntas académicas de ¿Para qué, qué evaluar y qué hacer con la información? cobran sentido tanto por su pertinencia histórica como heurística.

\section{¿Para qué evaluar?: las múltiples funciones de la evaluación}

En su trabajo sobre el pacto fiscal, la CEPAL establece una clara conexión entre el macro proceso del desarrollo de América Latina y el micro proceso de la evaluación de los resultados de la gestión pública. Los investigadores de la CEPAL sugieren que la agenda de la modernización 
del Estado, la reforma administrativa y la evaluación del desempeño de la gestión pública son pre-requisitos para promover una agenda de desarrollo que incluya estabilidad macroeconómica, mayor equidad, una asignación más efectiva de los recursos y un crecimiento económico sostenible.

Conceptualmente, este argumento tiene sentido: la gestión pública es la instancia mediadora entre la implementación de las políticas públicas formuladas en el campo de la política y los resultados que se obtengan de su implementación en el campo de la administración. El mejoramiento de la gestión pública cobra en este contexto una perspectiva estratégica de consolidación institucional: “....se trata de mejorar la productividad del gasto público, haciendo rendir más y mejor los fondos asignados a las diversas partidas del presupuesto del Estado, de modo que hasta los usuarios de servicios públicos más exigentes y los contribuyentes de impuestos más renuentes encuentren justificada la provisión gubernamental de bienes públicos y demás intervenciones del Estado en la economia" (p. 262).

Dicen los autores que una mejor gestión del gasto público mejora la imagen del Estado y aumenta su legitimidad; libera recursos que pueden ser aplicados a satisfacer las necesidades de los grupos de ingresos más bajos, aumentando así también la equidad; y finalmente mejora directamente los bienes y servicios, contribuyendo a aumentar el bienestar de la población y la productividad y competitividad del sector privado.

La racionalización del gasto público supone modernizar todas las áreas de la administración pública, incluyendo la gestión financiera y de recursos humanos. Ello requiere de una gestión estratégica, con una clara definición de visión, objetivos y metas, y con criterios e indicadores para poder evaluar el desempeño de las organizaciones y los programas a través de los cuales se implementan las políticas de gobierno.

La importancia política de la evaluación a este nivel macro coincide con el estado del arte de la gestión estratégica y la justificación de la evaluación como herramienta crítica en el nivel micro de la administración. La evaluación como técnica administrativa ayuda a mejorar la gestión porque produce la información necesaria para identificar y entender las causas de los logros y los fracasos o problemas del desempeño individual y colectivo, dentro de un contexto de planeación estratégica (Bryson, 1988).

Esta visión es evidente en los documentos del caso colombiano y, del caso chileno, aunque su importancia predomina más en el segundo. En las guías elaboradas por el Comité Interministerial (CIMGP) para entrenar a los funcionarios chilenos que participarán en sus iniciativas de modernización dicen: "Evaluar la gestión implica medir sistemáticamente, sobre una base continua en el tiempo, los resultados obtenidos por las principales unidades o departamentos de una institución, y comparar dichos resultados con lo deseado o planificado, a fin de elevar su eficiencia y productividad" (CIMGPa, 1996: 1). 
En relación con la importancia y necesidad de medir indicadores de gestión, la misma guía dice: "La evaluación cualitativa y cuantitativa del desempeño...permite detectar inconsistencias entre el quehacer de la institución y sus objetivos prioritarios, induce adecuaciones en los procesos internos y aporta mayor transparencia a la gestión pública. [...]Lograr una gestión más eficiente y comprometida con los resultados implica transformaciones importantes en el funcionamiento de las instituciones públicas" (CIMGPb, 1996: 1).

Efectivamente, bien utilizada, la evaluación promueve procesos de aprendizaje continuo que permiten refinar la capacidad humana e institucional para avanzar los objetivos planteados hacia el cumplimiento de la misión. Las minucias de este proceso están bien descritas en la guía para los funcionarios chilenos, que dice así: "Un sistema que entregue información regular sobre la calidad de la gestión posibilitará mayor eficiencia a la asignación de recursos físicos, humanos y financieros, proporcionará una base de seguridad y confianza en su desempeño al conjunto de los funcionarios implicados en su implementación — en la medida que delimita mejor el campo de sus atribuciones y deberes - , incrementará la autonomia y responsabilidad de los directivos - en tanto cuente con bases sustentables de información para la toma de decisiones, y ayudará a mejorar la coordinación con los demás niveles del aparato público" (CIMGPb, 1996: 1).

Todos estos aspectos técnicos obviamente se encuentran insertos en el caso chileno, al igual que en el colombiano, en un marco político. Ramón Figueroa, máxima autoridad oficial en asuntos de evaluación de la gestión pública en Chile durante el segundo gobierno de la Concertación, afirma: “¿Por qué debemos evaluar? [...] El principal objetivo es sustentar un sistema público que tenga credibilidad y que responda a las necesidades del público" (Figueroa, 1998: 209).

Efectivamente, hay una razón adicional por la cual es importante evaluar los resultados de la gestión en un contexto democrático. Al respecto, dice Pablo Abitbol en su reflexión sobre el sistema de evaluación diseñado en Colombia, que la evaluación de la gestión se puede considerar como una herramienta crítica de la modernización del Estado por su papel dentro del proyecto de construcción de una sociedad más equitativa y democrática. Respondiendo a la pregunta de qué significa evaluar en términos de resultados en este contexto, Abitbol afirma: "Ante todo, esta modernización es un proceso que abarca mucho más que la innovación técnica de las prácticas estatales. Modernizar el Estado significa desatar procesos orientados a la consolidación de una idea moderna del Estado (énfasis en el original), que, en términos generales, implica la construcción de espacios democráticos de toma de decisiones públicas" (p. 1, documento sin fecha, circa 1994). 
Más aún, Abitbol realza el carácter democrático de la evaluación del desempeño en el sector público al relacionar la doble obligación del Estado de responder a un mayor control ciudadano de las acciones del Estado y de formular objetivos que interpreten las preferencias de la ciudadanía: "La evaluación de resultados de la gestión pública... ofrece la posibilidad de articular la transparencia de las acciones del Estado al cumplimiento de objetivos, añadiendo claridad a las responsabilidades de cada unidad gerencial del gobierno y a la labor fiscalizadora que sobre ellas hace la sociedad civil" (p. 2, sin fecha).

Los diseñadores del sistema de evaluación en Colombia concibieron la evaluación de la gestión como la mejor herramienta posible para generar un cambio en la cultura de la administración pública colombiana, al pretender "abrir" la gestión al público, ofreciéndole amplia información generada por el sistema. El sistema serviría entonces para motivar y fortalecer en la práctica una gestión orientada a resultados, en la medida en que las acciones gubernamentales se hicieran públicas y los funcionarios tuvieran que rendir cuentas de una manera más transparente. Al respecto, dice Miguel Gandour (1996), uno de los primeros directores de la unidad encargada del proceso: "Un proceso de democratización lleva implícita la exigencia de hacer más pública la información referente a las ejecutorias del gobierno. El aumento del número de los procesos de toma de decisiones en los que la ciudadanía puede ejercer influencia, entraña el derecho de todos sus miembros a un mayor conocimiento de las acciones gubernamentales.[...] Este carácter público de las acciones gubernamentales no se refiere únicamente al acceso que tengan los ciudadanos a información sobre los resultados de la gestión, sino además a la transparencia de los procesos de toma de decisiones que conducen a esos resultados" (p. 27).

Para los gestores del sistema colombiano, el sistema de evaluación además de ser una herramienta administrativa moderna - era una respuesta a los mandatos políticos de la Constitución de 1991. El sistema de evaluación se propone como una herramienta para contribuir tanto a la modernización del Estado como a la democratización de la sociedad colombiana. En las palabras de Abitbol: "la modernización del Estado y la democratización del sistema social, político y económico son procesos complementarios y mutuamente constitutivos" (p. 2, sin fecha).

Estas reflexiones sugieren que la evaluación de la gestión pública cumple tanto funciones técnicas como funciones políticas y simbólicas que son muy importantes. En resumen, como respuesta a la pregunta ¿Para qué evaluar la gestión pública en América Latina?, podemos decir que ésta es una agenda importante porque la evaluación puede contribuir a fortalecer y profundizar la institucionalidad democrática si logra: 
- establecer claramente los varios niveles de responsabilidades de

los funcionarios públicos y su obligación de dar cuenta de sus acciones (accountability);

- garantizar una gestión más transparente y generar información sobre la eficiencia del uso de recursos;

- motivar a los empleados públicos a dar prioridad a la perspectiva del ciudadano y del usuario del servicio en el diseño de programas y servicios, y en el diseño de los instrumentos para evaluarlos;

- mejorar la calidad en los servicios prestados por el sector público, lo cual incide directamente en los dos pilares de una política de desarrollo: el bienestar de la población y la competitividad del sector productivo;

- mejorar la coordinación entre organizaciones públicas que trabajan en los distintos niveles del aparato público;

- mejorar la productividad del sector público, legitimando a los ojos de los usuarios y ciudadanos la provisión gubernamental de bienes públicos.

En teoría, a través de estas consecuencias, un sistema de evaluación contribuye a democratizar la gestión y a ejercer un control más racional del gasto público, fortaleciendo así la capacidad del Estado para gobernar. En este sentido, la evaluación de la gestión pública contribuye a la democratización de la sociedad y del Estado, a la consolidación de procesos de desarrollo institucional, y por tanto, al fortalecimiento de las instituciones democráticas.

Si bien un buen sistema de evaluación no es suficiente para lograr todo esto, sí es necesario para que ello ocurra. Pero la evaluación de la gestión pública no es tarea fácil. Por un lado, muchos aspectos del accionar público son intangibles, simbólicos o puramente conceptuales, y por tanto, es difícil encontrar indicadores adecuados para medirlos. Por ejemplo, el sector público debe equilibrar múltiples objetivos (eficiencia, equidad, estabilidad, crecimiento, transparencia, democracia) e intermediar intereses de diversos actores que compiten por lograr éstos con recursos generalmente escasos. De manera que la evaluación tiene que ser suficientemente sensible al impacto que estas negociaciones pueden tener en los resultados de la gestión pública. Ello supone concebir sistemas de evaluación cuyas características técnicas no reduzcan o amenacen la integridad de su naturaleza política.

Si la naturaleza de la evaluación de la gestión en el sector público va más allá de su carácter puramente administrativo, entonces los modelos de evaluación de la gestión pública no pueden ser simplemente aplicaciones mecánicas de los modelos del sector privado. Aunque no se trata de "reinventar la rueda", es importante que las aplicaciones se realicen con una clara conciencia del contexto político, histórico-social y cultural. 
Finalmente, la naturaleza misma de las actividades para implementar políticas públicas agrega una dimensión más a la complejidad de la evaluación de la gestión pública. En el sector público, el desempeño ocurre en varios niveles, que van desde el nivel personal (desempeño de los empleados) hasta el nivel de las políticas públicas de tipo nacional (desempeño de las políticas de gobierno), pasando por el nivel de los programas y las organizaciones. Estos niveles son interdependientes, pero cada uno de ellos representa un ámbito particular cuya gestión puede ser objeto de evaluación. Cómo hacer esto es, por sí mismo, un problema técnico y político de gran relevancia para la gestión pública.

\section{¿Qué evaluar y a qué nivel hacerlo?: el objeto y los niveles de la evaluación}

El estado del arte de la evaluación de la gestión pública sugiere que la evaluación debe orientarse principalmente a determinar si los resultados que se esperaban se han cumplido (Banco Mundial, 1996; Kettl, 2000). Esta orientación contrasta con otras que enfatizan, por ejemplo, la lógica de los procesos o de las teorías implícitas sobre intervención social. Los ejemplos presentados en la sección anterior sugieren que tanto el caso chileno como el colombiano se caracterizan por tener esta orientación.

En Colombia, el énfasis en los resultados está plasmado en el mismo nombre del sistema, SINERGIA. Además de tener un sentido metafórico, el término SINERGIA representa una abreviación al nombre asignado al sistema: Sistema Nacional de Evaluación de Resultados de la Gestión Pública. Respondiendo a la pregunta de ¿Para qué se evalúa? Silvia Escovar (1996), una de las primeras directoras de la unidad responsable de la creación del sistema, afirma categóricamente: "Sinergia ha sido concebido como una herramienta gerencial para el mejoramiento de la gestión pública. Esto implica acercar los resultados de las acciones de gobierno a lo pretendido en la planeación. Por lo tanto, la utilidad de la evaluación depende directamente de su contribución al logro de resultados de dichas acciones" (p. 14).

En Chile, Figueroa (1998) articula esta misma dirección cuando afirma que "Los dos gobiernos de la Concertación se han orientado a medir y evaluar resultados, y su principal preocupación ha sido evaluar el impacto de las acciones del gobierno en la gente" (p. 209).

Por lo general, el objeto de la evaluación a nivel organizacional incluye medidas del desempeño en términos de por lo menos cuatro dimensiones de la gestión: economía (capacidad para movilizar recursos y costo/beneficio), eficiencia (costos considerando los productos), eficacia (cumplimiento de los objetivos) y calidad (la experiencia del servicio por parte del usuario). Estas son, efectivamente, las dimensiones de gestión que el sistema de 
medición implementado en las iniciativas de modernización chilenas se propone evaluar (Waisbluth, 1998). Para medir estas dimensiones de la gestión, las guías chilenas proponen crear indicadores a los objetivos formulados en cada dimensión, considerando por separado la gestión de procesos, de productos (o servicios) y de impactos (Dirección de Presupuesto, 1999). Se espera, así, que los sistemas de medición usados permitan determinar en qué medida los recursos, los procesos y las actividades realizadas, así como las estrategias implementadas para cumplir los objetivos propuestos, contribuyen de una manera concreta para avanzar en la misión de la agencia en cuestión.

Estos conceptos, derivados directamente de la metodología de la Planeación Estratégica, son aplicados de una manera bastante fidedigna, con las adaptaciones del caso, tanto en las técnicas de medición que proponen las guías chilenas para participar en las iniciativas de modernización, como en las guías colombianas para instruir a los funcionarios en la metodología necesaria para construir sus informes. Sin embargo, hay una diferencia entre los dos casos, y es que en Colombia, uno de los requisitos para la elaboración de planes con sus respectivos indicadores es de mostrar de qué manera las metas y objetivos propuestos por cada organización se relacionan con el Plan de Desarrollo del gobierno. Esto agrega un nivel de complejidad al trabajo de los funcionarios de cada organización, pero a la vez, da mayor coherencia política a la información recogida a través del sistema.

Este énfasis en los macro procesos representa un esfuerzo por “empujar" la teoría de la gestión estratégica tradicional, ajustándola así a los requerimientos de la administración pública. Si la gestión pública moderna debe estar orientada a resultados más que a procedimientos, y si su evaluación debe reflejar esta orientación, ¿cómo abordar el problema de la gestión del desempeño cuando éste tiene implicaciones que van más allá de resultados medibles en términos financieros? Más aún, en el sector público, el desempeño de las organizaciones se relaciona con el nivel de las políticas públicas y los programas para implementarlas, que normalmente se desarrollan en varias organizaciones o cruzan varias jurisdicciones.

De allí la importancia de considerar varios niveles de la gestión en un sistema de evaluación. En el nivel más micro de la gestión, tenemos la evaluación del desempeño de los empleados que participan en una organización pública para cumplir su misión. Esta es una actividad que compete al ámbito de la función gerencial denominada "gestión de recursos humanos". Un nivel intermedio de evaluación del desempeño (nivel meso) corresponde a la evaluación de las organizaciones públicas encargadas de proveer servicios o implementar aspectos concretos de los programas de gobierno. La evaluación del desempeño organizacional es una actividad que compete al ámbito de la función gerencial de la planeación estratégica. 
A un nivel macro está la evaluación del desempeño de los programas que cruzan varias organizaciones, y un cuarto nivel, el más macro, se refiere a la evaluación del desempeño del gobierno como conjunto de instituciones comprometidas con una serie de políticas públicas que se han gestado democráticamente y por tanto representan un pacto con los ciudadanos.

Como dijimos antes, estos niveles son interdependientes, pero cada uno de ellos representa un ámbito particular cuya gestión puede ser objeto de evaluación. Para efectos de este trabajo, consideraremos los niveles tres y cuatro dentro de un mismo grupo. Estos dos niveles macro corresponden a actividades que competen a entidades supra-organizacionales, encargadas por ley o por mandato ejecutivo de asegurarse del cumplimiento de los mandatos y el uso efectivo de los recursos públicos.

Cada uno de estos niveles tiene y requiere mediciones de distintos tipos y probablemente ciclos diferentes de evaluación. Por ejemplo, la evaluación de las políticas públicas requiere un horizonte más amplio que la evaluación de los objetivos de cada organización y que las evaluaciones del personal en cada una de ellas. Pero a la vez, estos niveles, más que independientes, son interdependientes.

Si se quiere hablar de un sistema, se tiene que hablar necesariamente de las interconexiones y, sobre todo, de la articulación de estos niveles. Por ejemplo, la evaluación del desempeño de un funcionario (nivel micro) tiene que estar referida a la manera como sus acciones posibilitan el cumplimiento de la misión organizacional (nivel meso); y la evaluación de la organización tiene que estar referida a la manera como ésta posibilita acciones del gobierno para implementar las políticas públicas (nivel macro). De esta manera, los niveles más macro de la evaluación pueden utilizar información de los niveles inferiores, e igualmente, los sistemas de los niveles inferiores pueden ser ajustados y desarrollados con la información que se recibe de los niveles superiores.

Este fue, en efecto, el espíritu que guió el desarrollo del sistema nacional de evaluación colombiano, SINERGIA. Durante su gestación, la pregunta crítica fue cómo articular los diversos niveles, y más importante aun, cómo asegurar que los usuarios del sistema pudieran establecer comparaciones válidas entre organizaciones tan diversas como el Ministerio de Defensa y el Ministerio de Educación, por ejemplo. Como respuesta, se crearon los Planes Indicativos, instrumentos que deberían ser elaborados por cada entidad y presentados al DNP para efectos del análisis, comparación y publicación de la información presentada en ellos. El DNP utilizaría también la información como base para la toma de decisiones de ajuste de las políticas públicas y asignación de los rublos del presupuesto (Ospina, 1997).

El sistema funcionaría de la siguiente manera. Con el Plan Indicativo, las agencias establecerían metas y objetivos para los cuatro años 
correspondientes a cada período presidencial. Estas metas se formularían en relación con su misión, pero también con referencia al Plan Nacional de Desarrollo elaborado por el gobierno para el mismo período. Los planes indicativos, por tanto, partirían de las políticas de desarrollo nacional y especificarían los productos e impactos, las actividades para alcanzarlos y los indicadores con los cuales cada organización se comprometía a evaluar su desempeño. Sus contenidos serían especificados conjuntamente por miembros de cada entidad con el apoyo de miembros de las unidades especializadas en el Departamento Nacional de Planeación (DNP).

Cada Plan Indicativo se actualizaría anualmente con base en autoevaluaciones internas entregadas al ministerio correspondiente, y éste a su vez las entregaría al Departamento Nacional de Planeación. Además, el Departamento conduciría cinco o seis evaluaciones ex-post en algunas organizaciones y programas seleccionados estratégicamente. El DNP produciría un informe anual para el Congreso con un resumen de todas las evaluaciones y con un análisis del progreso alcanzado para el cumplimiento del Plan Nacional. Produciría además un documento estratégico con los resultados de las evaluaciones y propondría los ajustes necesarios a nivel de programas y políticas.

De ser aprobado por el Congreso, éste se convertiría en el documento orientador del trabajo para todas las entidades. Esta información, junto con el borrador de los planes indicativos para el año siguiente, servirían como base para asignar el gasto público y los rubros presupuestales correspondientes. La información permitiría, además, identificar necesidades de desarrollo institucional y ajustar la formulación estratégica de las políticas públicas. En teoría, se esperaba también que las auto-evaluaciones y el documento del DNP servirían como fuentes de aprendizaje y mejoramiento al interior de cada entidad (Banco Mundial, 1997).

Por la localización institucional de los funcionarios que lo crearon y por el mandato que lo inspiró, el diseño de SINERGIA refleja un énfasis en el nivel más macro de la evaluación, el nivel de los programas y políticas de gobiemo. En este sentido, el sistema surge como una política de arriba hacia abajo, donde la lógica es impuesta desde la Planeación Nacional hacia las organizaciones y funcionarios. Es posible que esto explique en parte la falta de atención a los sistemas de evaluación del desempeño de los empleados y a cómo éstos se articularían a los niveles meso y macro del sistema. En efecto, el sistema como tal no contempla el nivel individual del desempeño, dejando a otras entidades la tarea de abordar el problema de la evaluación de los empleados, el problema de los incentivos asociados a ésta, y otros aspectos de la gestión de los recursos humanos.

Esto representa una debilidad del sistema colombiano. Incluso aceptando la idea de una división del trabajo entre varias organizaciones 
públicas para evaluar los distintos niveles del desempeño, el problema se

evidencia en la ausencia de un mecanismo para asegurar la coordinación entre estas instancias. En este sentido, el sistema está integrado verticalmente solamente hacia arriba (al articular el problema del desempeño organizacional con el de las políticas públicas), pero carece de un grado importante de integración hacia abajo (ignorando el nivel micro de la evaluación del personal). Más aún, carece también de coherencia "horizontal" por no estar bien articulado con las otras entidades encargadas de regular la evaluación del desempeño individual.

En el caso chileno, aunque la evaluación también se concibe en referencia a la modernización como política de gobierno, la atención hacia el problema de la medición de los resultados de la gestión parece haberse puesto desde un comienzo en los niveles micro y meso, o sea en la evaluación del desempleo individual y organizacional. Por ejemplo, Figueroa (1998) ofrece la siguiente definición de la evaluación del desempeño: "Cuando hablamos de evaluación del desempeño nos referimos al conjunto de mecanismos o elementos que permiten definir el grado en que las personas contribuyen al logro de los estándares u objetivos de una institución" (p. 209).

Este énfasis en el nivel de las personas y las organizaciones se ve reflejado en la manera como el sistema de medición de la gestión pública en Chile está jalonado por la agenda de mejorar la administración pública (como parte del esfuerzo de modernización), más que por la agenda para evaluar las políticas de gobierno. En contraste con Colombia, en su primera etapa al sistema chileno parece hacerle falta una mayor integración vertical hacia arriba, mientras que su fortaleza está en la integración hacia abajo.

Por ejemplo, los Programas de Mejoramiento de la Gestión (PMG) son compromisos institucionales para que un servicio público genere un esfuerzo por aumentar cualitativa o cuantitativamente "respecto de los resultados históricos o [para] innovar hacia nuevos logros" (Dirección de Presupuesto, 1999: 1). Los PMG están basados en un sofisticado sistema de medición de indicadores para documentar el cumplimiento de los compromisos. Este sistema se consolidó a partir de un proceso de negociación con la asociación de empleados públicos, para articular las estrategias de evaluación del desempeño individual con las del desempeño organizacional (Dirección de Presupuesto, 1999). El proceso culminó con la creación de una ley que manda establecer incentivos para los funcionarios de los servicios que demuestren el cumplimiento de los objetivos contenidos en su Programa de Mejoramiento.

En Chile, el énfasis en la evaluación se desarrolló incrementalmente alrededor de proyectos piloto de indicadores de gestión implementados en algunas instituciones seleccionadas (Plan General). Los proyectos iniciales 
eran parte de un esfuerzo "modernizador" para aplicar la metodología de la planeación estratégica al sector público. El proceso de implementación fue gradual. Inicialmente se impulsó a partir de los convenios de desempeño, que eran voluntarios. Más adelante se desarrolló la iniciativa de los Programas de Mejoramiento de la Gestión, PMG, a través de la cual sus gestores esperaban eventualmente poder comparar el desempeño de las distintas agencias. En este sentido, en Chile existe un sistema de medición de la gestión que genera información para su mejoramiento, pero no existe un sistema nacional de evaluación, como en el caso de Colombia.

Efectivamente, en el caso de Chile el énfasis en la evaluación parece surgir para apoyar un conjunto de iniciativas que son parte de la agenda de modernización de la gestión pública, más que de una agenda o mandato independiente para evaluar la gestión. Esta es una distinción sutil pero importante. El sistema de medición chileno es una herramienta más en la agenda de modernización, definida esta agenda como un "conjunto de instrumentos de gestión y evaluación orientados a un proceso continuo de perfeccionamiento de la gestión de las instituciones públicas" (Dirección de Presupuesto, 1999: 1).

Esta agenda consta de diversas estrategias en el ámbito ministerial, interministerial y de los servicios públicos, y busca desarrollar cinco líneas de acción, de las cuales solamente una enfatiza en los resultados (las otras son: la orientación al usuario, la visión estratégica, la participación de los funcionarios y la desconcentración de los ministerios y servicios). Las principales estrategias de la agenda modernizadora incluyen los Convenios de Desempeño, los Compromisos de Modernización, y más recientemente, los Programas de Mejoramiento de la Gestión (PMG). Por ejemplo, los Compromisos de Modernización entre cada servicio público y el Presidente de la República, creados para promover iniciativas modernizadoras en las entidades públicas, utilizan indicadores de gestión para determinar su cumplimiento y para generar balances de gestión, en teoría comparables entre si. Los indicadores y los balances de gestión representan el componente de evaluación del esfuerzo modernizador.

Solamente con los PMGs el proceso de evaluación de la gestión en Chile empieza a adquirir carácter más integral e intenta convertirse en un esbozo de sistema de evaluación, sin lograrlo del todo. Ciertamente los PMGs introducen el tema de la evaluación al discurso legislativo, a partir de la aprobación en el Congreso de los incentivos para los funcionarios. Además, estos programas promueven un mayor nivel de articulación con los niveles macro del desempeño, porque involucran en el proceso tanto a las agencias como a los ministerios al cual pertenecen y al gobierno central. La guía desarrollada para presentar un PMG describe el proceso de la siguiente manera: "Una vez planteada la misión, identificados los productos/ usuarios, definidos los objetivos estratégicos (de mediano y largo plazo), 
formulados los objetivos de gestión, priorizados (como expresión anual del logro), determinados los indicadores, el Jefe de Servicio se compromete formalmente ante el Ministro del ramo a un mejoramiento del desempeño institucional; el Ministro, por su parte, asume un compromiso sectorial ante las autoridades centrales" (p. 18).

Más aún, el compromiso entre el Ministro y el gobierno central se formaliza a través de un decreto. Pero la articulación entre los niveles micro y meso con el nivel macro se concibe como algo implícito, que ocurre automáticamente como el resultado de la sumatoria de los esfuerzos de modernización. Esto representa una debilidad del sistema chileno. Por ejemplo, la guía incluye en las instrucciones la siguiente sugerencia: "Con el objetivo de agilizar el proceso de tramitación de los decretos, se sugiere elaborar un decreto por cada Ministerio que contenga los programas de mejoramiento de los servicios relacionados o dependientes" (p. 19). Pero nótese que la razón que se da no tiene que ver con la integración ni horizontal ni vertical, sino con la necesidad pragmática de tener menos tramitación. Este pragmatismo es característico de la propuesta de evaluación de la gestión en el caso chileno y puede tener consecuencias negativas en el largo plazo.

A diferencia del caso colombiano, en Chile parece haberse dado un proceso que empezó de abajo hacia arriba en lo que se refiere a la preocupación con los niveles de la evaluación del desempeño. Inicialmente, los esfuerzos de evaluación se promueven al interior de cada organización y sólo más tarde empiezan a buscarse maneras para ubicar cada proceso dentro de un ámbito común. En efecto, según documentos oficiales, el gobierno chileno se encuentra actualmente involucrado en un proceso para buscar mecanismos que permitan integrar las metodologías e instrumentos de evaluación del desempeño que se usan en cada entidad, y para unificar en un sistema integrado lo que se hace en cada organización, puesto que hasta el momento ha sido difícil establecer comparaciones.

\section{¿Cómo asegurar el uso de la información?: de la medición a la gestión del desempeño}

- La existencia de un sistema - bien sea de medición o de evaluación - por sí solo no garantiza el mejoramiento de la gestión pública, ni mucho menos su contribución para fortalecer la democracia. La viabilidad del sistema para promover una cultura orientada a resultados y para ayudar a fortalecer las instituciones públicas depende en gran parte del uso que se le dé a la información generada.

Expertos en evaluación de programas públicos hacen una distinción entre los términos "medición del desempeño" y "gestión del desempeño" 
(Rist, 1990; Smith y Barnes, 1998). El primer concepto se refiere a la creación de un sistema de indicadores y herramientas que permiten medir el rendimiento de un individuo, organización o programa. El segundo se refiere al uso de la información generada por este sistema de indicadores para aprender y tomar decisiones a un nivel más estratégico. La gestión del desempeño está directamente relacionada con la idea de la misión organizacional y ayuda a determinar qué áreas necesitan mejorar para su cumplimiento; mientras que la medición del desempeño es el instrumento técnico para lograr ese objetivo.

La diferencia entre medición y gestión del desempeño radica en el aprendizaje generado cuando los indicadores del desempeño se utilizan de una manera coherente y sistemática para tomar decisiones con respecto al individuo, organización o programa del cual se ha obtenido la información. Aunque existen muchos sistemas de medición, pocos se convierten en herramientas de gestión, porque la medición no produce automáticamente mejores resultados (Smith y Barnes, 1998).

Por ejemplo, a pesar de que la metodología para crear indicadores de gestión lleva ya más de cincuenta años de experimentación en los Estados Unidos, el uso de la información sigue siendo el punto débil de esta tecnología gerencial. En efecto, cuatro años después de implementar la legislación que en 1993 exigió a las agencias federales producir un plan estratégico, metas anuales de desempeño e indicadores para demostrar progreso, la Oficina de Evaluación de este gobierno concluye en 1997 que la información no es usada para tomar decisiones en los programas pertinentes. Situaciones similares se han documentado a nivel de varios estados y municipalidades estadounidenses (Caiden y Caiden, 1997).

¿Cómo motivar a los gerentes y empleados para que usen la información? ¿Qué tipo de incentivos es necesario crear para que la evaluación no se convierta en un simple ejercicio burocrático sino que, por el contrario, cumpla su papel administrativo y político dentro de una agenda más amplia? El problema no es solamente de tipo técnico sino también humano y político.

Algunos elementos de la información recogida en Chile y Colombia sugieren la importancia de estas preguntas. Por ejemplo, una característica distintiva del caso chileno es el éxito de los convenios personalizados para motivar los procesos de mejoramiento y para institucionalizar la evaluación de la gestión como una herramienta gerencial. Esta característica refleja una clara influencia de la filosofía de la Nueva Gestión Pública pero adaptada a las idiosincrasias de la región. El énfasis en la filosofía de los contratos o acuerdos que incentivan directamente al gerente combina algo del estilo de Nueva Zelanda - al considerar a los gerentes como "agentes" y el gobierno como "principal”, pero sin formalizar esta relación sino dejándola como un contrato moral - y algo del estilo pragmático estadounidense, 
con su fuerte tradición de gestión del desempeño y la aplicación formal de técnicas de medición del mismo. La aplicación de estrategias con esta lógica se entiende en parte por la fuerte tradición de estabilidad institucional y de respeto a las instituciones políticas que han caracterizado a Chile a lo largo de su historia.

En Colombia también trataron de implementar los Acuerdos de Eficiencia, que representaban compromisos políticos formales de cada Ministro con el Presidente. Estos se propusieron como acuerdos sustentados con metas y objetivos concretos (formulados en los planes indicativos), que buscaban articular formalmente el compromiso del ministerio para contribuir al cumplimiento del Plan de Desarrollo. Se esperaba así dar mayor importancia a los planes indicativos y hacer que la alta directiva de cada organización se involucrara personalmente en el proceso de evaluación, promoviendo de esta manera una cultura gerencial orientada a los resultados.

Sin embargo, las circunstancias políticas de creciente ingobernabilidad que caracterizaron la coyuntura bajo la cual se empujó la idea de los Acuerdos impidieron que estos se institucionalizaran. En un contexto de alta turbulencia política, los planes indicativos, que eran el pilar del sistema de medición utilizado por SINERGIA, se volvieron elementos amenazantes para la alta gerencia, y quedaron relegados a una actividad burocrática, poco o escasamente articulados a la visión del ministro y su equipo de trabajo (Ospina, 1997). La coherencia teórica del modelo propuesto desde Planeación Nacional no representó un incentivo poderoso para los ministros, quienes tenían problemas más inmediatos que resolver, particularmente dada la incertidumbre y poca legitimidad del ejecutivo para gobernar en ese momento. ${ }^{2}$

Estas observaciones sugieren que para que los sistemas de medición se conviertan en sistemas de gestión, además de indicadores y mediciones técnicamente elaboradas, se necesitan mecanismos organizacionales e incentivos institucionales que comprometan personalmente a los empleados y gerentes en agendas que vayan más allá de las fronteras de cada organización. En parte, el desafío está en generar una dinámica que motive a los líderes de una organización pública a demandar y usar información de una manera explícita y sistemática para tomar decisiones que son parte de una agenda no sólo gerencial sino política.

El problema del uso de la información apunta nuevamente a la articulación de los niveles micro, meso y macro como elemento crítico de un sistema de evaluación que busque ser más que una herramienta técnica. El uso de la información representa el elemento más político de la evaluación de la gestión pública. Los casos de Chile y Colombia son muy jóvenes y se necesita más investigación de campo para aprender de ellos. Dos ejemplos documentados en los Estados Unidos ilustran algunos aspectos de esta dinámica que vale la pena resaltar. 
El caso del Departamento de Policía en la ciudad de Nueva York es ilustrativo de la importancia de los mecanismos organizacionales para promover el uso colectivo de la información y las consecuencias que ello conlleva para el mejoramiento de la gestión. El proceso empezó con la modernización del sistema de computadores para recoger información y con la creación de un sistema de indicadores de gestión para que los comandantes de los precintos demostraran el progreso de su trabajo. Sin embargo, a pesar de la energía invertida en la recolección de los datos, nadie los utilizaba (Smith, 1997).

Esto cambió cuando los comandantes de los precintos fueron "invitados" a rendir cuentas sobre el progreso de su jurisdicción. Levando los datos a una reunión de alto mando en la oficina central de la policía, presidida por el Comisionado. Utilizando grandes pantallas que todos podían leer, el grupo empezó a monitorear el progreso con tablas y gráficos que mostraban de una manera comparativa el desempeño de cada precinto.

Con la transferencia de la rendición de cuentas del Comisionado a los comandantes de cada precinto sobre el resultado de la gestión, la información pronto empezó a fluir en ambas direcciones (Smith y Barnes, 1998). Los comandantes empezaron a exigirle a sus subalternos mejores datos, quienes a su vez pusieron presión hacia abajo hasta influenciar los niveles más bajos de la pirámide, o sea a los policías en la calle. Todos empezaron a usar los datos para analizar tendencias significativas en relación con la actividad criminal y la actividad policial en su zona. Empezaron también a buscar soluciones a los problemas identificados, ubicando éstos dentro del plan estratégico propuesto desde la administración central.

Pronto las reuniones en la oficina central se convirtieron en oportunidades para intercambiar ideas, problemas y soluciones que empezaron a ser usadas por todos. El mayor flujo de información horizontal redundó en una mejor coordinación interdepartamental, y con el tiempo, la reunión se convirtió en una instancia para tomar decisiones estratégicas a nivel ejecutivo. Esta dinámica generó una nueva manera de entender la misión y las tareas críticas de la organización, con el desarrollo de estrategias integradas y con un mayor nivel de coordinación del trabajo (Smith, 1997).

El crimen en Nueva York se ha reducido considerablemente en los últimos años, y la policía atribuye esto, en parte, al nuevo uso de la información para la toma de decisiones estratégicas. Existen debates sobre la validez de este argumento, dado que la reducción del crimen es un fenómeno nacional, y no se han realizado evaluaciones sistemáticas del impacto de la iniciativa descrita. Se necesita más investigación empírica para determinar la contribución de estos procesos al mejoramiento de la gestión pública (Caiden y Caiden, 1997). Pero a primera vista, la evidencia sugiere que un enfoque de gestión por resultados, con un compromiso formal por parte de los gerentes, con incentivos y mecanismos 
organizacionales dirigidos a mostrar su utilidad, y con una visión orientada a solucionar problemas para mejorar los servicios, puede tener implicaciones directas en el mejoramiento de la gestión pública. Además, la evaluación juega un papel clave en el éxito de este proceso.

Si el caso de la Policía de Nueva York enfatiza la función técnica del uso de la información para mejorar la gestión y volvería más estratégica, el siguiente caso enfatiza la función política del uso de la información al ilustrar un proceso de concertación que involucró a la ciudadania en la evaluación de la gestión.

El caso se refiere al trabajo realizado en el área de la provisión de servicios sociales en el estado de Vermont, en los Estados Unidos (Hogan, 1999a). En este estado se han creado una serie de colaborativos a través de los cuales fundaciones, agencias públicas y ONGs desarrollan conjuntamente una visión común sobre el bienestar social de la población. Los miembros de estas organizaciones se han comprometido a usar esta visión como referencia para su trabajo. De ella se derivan indicadores concretos que representan mediciones del impacto del trabajo y están referidos no solamente a la misión formulada para el área social en el estado en su conjunto, sino además a la misión de cada organización que se compromete a trabajar por ellos. Este estilo de trabajo también ha generado integración horizontal, ya que algunas agencias públicas se han unido en alianzas estratégicas para establecer metas y objetivos comunes en relación con el avance de indicadores selectos relacionados con alguno de los aspectos de la visión (Hogan, 1999).

Esta visión fue formulada a través de un proceso de concertación liderado por el equipo de alta gerencia de la Agencia de Servicios Humanos y del Departamento de Educación, en el cual participaron los diferentes actores interesados, incluyendo miembros de la comunidad. Del proceso surgió una lista que guía la acción de los empleados y gerentes de cada organización. La lista presenta los resultados esperados si la acción concertada de todos pudiera hacer realidad esta visión. Estos resultados se formularon como proposiciones que describen el impacto que debería tener el trabajo conjunto de las organizaciones en el bienestar de las poblaciones "vulnerables" de Vermont. De acuerdo con esta visión, se aspira que algún dia en el estado de Vermont sea posible decir que: las familias, los jóvenes y los individuos participan y contribuyen a la toma de decisiones y actividades públicas en su comunidad; las mujeres embarazadas y sus bebés recién nacidos prosperan; los infantes y los niños crecen sanos; los niños están preparados para ir a la escuela; los niños tienen éxito en la escuela; los niños viven en familias estables, las cuales cuentan con suficiente apoyo; los jóvenes escogen comportamientos sanos; los jóvenes realizan una transición exitosa hacia la adultez; los ancianos y las personas minusválidas viven con dignidad e independencia 
en los lugares que ellos prefieren; y las familias y los individuos viven en comunidades seguras y se sienten apoyados por éstas (Hogan, 1999a).

El esfuerzo se considera exitoso porque los indicadores de estos impactos parecen mostrar una tendencia positiva en la dirección esperada en los últimos cinco años. Por ejemplo, en relación con el impacto \# 3, los indicadores sobre abuso y negligencia infantil muestran una reducción del $33 \%$ desde 1992, y el indicador del porcentaje de niños de dos años que están totalmente vacunados creció en un 17\% entre 1994 y 1995 (Hogan, 1999a).

Lo interesante de este modelo es que cada organización y cada colaborativo de organizaciones desarrolla sus propias estrategias, metas y objetivos para lograr estos resultados, pero todos se comprometen a trabajar por ellos. La evaluación del desempeño organizacional se realiza en cada organización de manera independiente, pero a la vez, el estado realiza una evaluación que busca documentar el progreso del trabajo conjunto. En efecto, el estado publica cada año un Informe de Bienestar, con 51 indicadores distribuidos entre los impactos esperados, con análisis de los datos y con comparaciones frente a las tendencias nacionales. Al mismo tiempo, se publican también Perfiles de la Comunidad, en donde la comparación se hace en relación con las tendencias del estado.

Este ejemplo sugiere entonces que es crítico formular muy explícitamente una visión de lo que los funcionarios y otras personas involucradas en la prestación de los servicios sociales se atreven a soñar. A partir de ella se crean objetivos e indicadores más concretos y de carácter intermedio para medir los impactos deseados. Así, el éxito se evalúa en la medida en que las metas y objetivos alcanzados ayuden a acercar la realidad a la visión formulada. Los indicadores de impacto, por su parte, que requieren un horizonte de medición más largo, sirven como estándares para generar aprendizaje en relación con los esfuerzos realizados en el mediano plazo, en el nivel macro de la gestión.

Los esfuerzos descritos se han realizado en jurisdicciones muy particulares (Nueva York representa una ciudad sui generis, y Vermont, un estado pequeño). Qué tan factible es extrapolar estas ideas para transplantarlas a otros contextos es una pregunta que está fuera del alcance de este trabajo. Pero su descripción sirve para ilustrar lo que significa organizar una agencia pública o un conjunto de ellas alrededor de una cultura de gestión orientada a resultados, y el papel que los sistemas de medición y evaluación cumplen en el proceso de mejoramiento continuo y aprendizaje que se genera con el uso de la información en todos los niveles, micro, meso y macro. 


\section{Conclusiones}

En este trabajo he presentado una conceptualización del fenómeno de la evaluación de la gestión pública alrededor de tres preguntas clave ¿para qué evaluar, qué evaluar y cómo asegurar el uso apropiado de la información? - , analizando aspectos selectos de esfuerzos de promoción de una gestión orientada a resultados en dos países latinoamericanos. Ello permitió clarificar el alcance del término "evaluación de la gestión pública" en Chile y Colombia, y reflexionar sobre su importancia en la gestión pública latinoamericana, resaltando algunos dilemas y oportunidades en la implementación de esta práctica como herramienta de modernización del Estado y consolidación de la democracia.

Es posible sacar algunas conclusiones tentativas de este ejercicio, a partir de la comparación entre los casos empíricos y de las reflexiones generadas con base en su discusión. Desde el punto de vista de la Administración Pública como disciplina, la evaluación de los resultados de la gestión pública se puede abordar desde tres niveles analíticos diferentes: un enfoque macro, que considera el desempeño del gobierno en la implementación de sus políticas públicas; un nivel meso, que considera el desempeño de las organizaciones públicas; y un nivel micro, que considera la contribución producida por el desempeño de los empleados al cumplimiento de la misión.

Un sistema de evaluación de la gestión pública supone un diseño institucional que produzca los incentivos necesarios para que sus usuarios vean la necesidad de acceder a la información de los tres niveles y de establecer conexiones entre éstos, creándose así una coherencia en las prácticas de los distintos niveles de la administración pública. Si el esfuerzo de evaluación se limita a generar indicadores de gestión en los niveles micro y meso de la acción, es posible hablar de un sistema de medición de la gestión que genera aprendizajes para mejorar las prácticas administrativas, mas no necesariamente para mejorar la calidad de las políticas públicas. Por otro lado, un sistema que se limite a generar indicadores en los niveles meso y macro, descuidando su articulación con el nivel micro de la acción gerencial, corre el peligro de perder legitimidad frente a quienes deberán implementarlo en la práctica, los gerentes y empleados.

En relación con las conclusiones empíricas, aunque no se ha buscado evaluar la validez de estos dos esfuerzos, ni de determinar si alguno de ellos es mejor que el otro, es claro que ambos representan esfuerzos muy valiosos para el desarrollo de la agenda de la modernización del Estado. Ambos proporcionan excelentes lecciones para avanzar esta agenda en la región. La comparación permite ilustrar los desafíos para crear sistemas de medición y evaluación modernos y viables en el contexto público. Además, los dos casos presentados en este trabajo sugieren que el diseño 
e implementación de esfuerzos sistemáticos de evaluación de la gestión pública tienen su propia dinámica em cada contexto nacional. En efecto, estos esfuerzos tienen diferencias importantes, tanto en su naturaleza como en su estructura y en los términos del proceso de implementación.

En Colombia, un mandato constitucional es el punto de partida para organizar un sistema de alcance nacional; una entidad técnica altamente especializada e independiente del Presidente asume el liderazgo y diseña un sistema de evaluación flexible pero, en teoría, unificado para todas las entidades. Una vez elaborado el sistema, se empiezan a desarrollar planes piloto con entidades de algunos ministerios para ajustar el modelo a la realidad y para comenzar a "venderlo". Sin embargo, en la práctica, a pesar de ser un mandato legal y a pesar de la excelencia del modelo en el papel, no se crearon los mecanismos formales ni existían los incentivos políticos para garantizar el uso del Plan Indicativo en las entidades, ni se dieron tampoco las condiciones para involucrar seriamente en el proceso a la alta gerencia de las entidades ni a los ministros de cada ramo.

En contraste, en Chile el ejecutivo crea un conjunto de indicadores de gestión insertados dentro de una estrategia articulada de modernización. En este país, el mandato legal se da después de la experimentación inicial, utilizando un proceso voluntario incentivado por acuerdos y convenios que involucran a la alta gerencia de cada entidad de una manera personal; una organización técnica también ayuda a jalonar el proceso (una oficina del Ministerio de Hacienda), pero con el liderazgo de una entidad muy cercana al Presidente, que además incorpora en su estructura de gobierno a todos los ministros (el Comité Inter-ministerial). En Chile, además, el proceso de integración solamente ocurre después de que el sistema parece funcionar en la práctica en cada organización que voluntariamente se ha acogido a las iniciativas, y este orden del proceso genera nuevos desafíos para su implementación. Finalmente, en el caso chileno, el esfuerzo incluye esquemas de bonificaciones por desempeño institucional, las cuales están totalmente ausentes en el caso colombiano.

El contraste entre los dos casos es muy interesante en términos del tipo de sistema que resultó a partir de cada proceso. En ambos casos es posible encontrar una fuerte influencia de los principios filosóficos y teóricos de la Nueva Gestión Pública, pero con resultados bastante disímiles. En Colombia emerge un sistema nacional de evaluación que tiene una gran coherencia y sofisticación teórica, como producto de un esfuerzo de conceptualización prolongado y liderado por un equipo multidisciplinario de expertos ubicados en una entidad técnica. Esto produce dos características que vale la pena resaltar como fortalezas potenciales del sistema: una es la excelente articulación entre los niveles meso de gestión y los niveles macro de las políticas públicas; la otra es la capacidad del sistema para establecer comparaciones entre los resultados de entidades cuya naturaleza es muy diferente. 
En teoría, el diseño del sistema es impecable. Pero a la vez el sistema ha tenido problemas de implementación, que algunos relacionan precisamente con su grado de sofisticación y con la falta de atención a la articulación entre los niveles meso y micro de la gestión. Otro factor importante es la coyuntura política dentro de la cual se creó e implementó el sistema (Ospina, 1997). Este entorno, caracterizado por un alto nivel de ingobernabilidad e incertidumbre, hizo que la alta gerencia no se atreviera a asumir riesgos, dado que su atención estaba dirigida a otras prioridades ajenas a la evaluación de la gestión, que de por si es un fenómeno amenazante (Kettl, 2000).

En contraste, en Chile el esfuerzo se basó en una aplicación pragmática de las técnicas de administración privada aplicadas al sector público con miras a modernizar sus instituciones, y en un proceso de conceptualización rápido y sin gran elaboración teórica más allá de la relacionada con los principios generales de la gestión. El resultado es un sistema de medición articulado a un programa de modernización. No es un sistema nacional de evaluación porque aún carece de herramientas que permitan establecer comparaciones precisas entre el desempeño de las distintas entidades y porque no está referido directamente al problema del desempeño de las políticas públicas. Por lo menos no todavía. En el caso de Chile, la evaluación busca mejorar las organizaciones públicas y el desempeño de sus empleados, o sea la administración pública, independientemente de los contenidos de los programas de gobierno. El gran desafío en el caso chileno estará en la etapa que sigue, cuando el esfuerzo de integración y unificación de las estrategias y herramientas de evaluación empiece a generar resistencias en las organizaciones que hasta ahora han operado independientemente, y cuando se establezcan las bases para que el sistema de medición se convierta en un sistema de evaluación de naturaleza nacional.

Por otro lado, la fortaleza del esfuerzo chileno radica en una clara articulación entre los niveles de desempeño organizacional y los niveles de desempeño del personal, puesto que ella ha permitido generar aprendizaje individual y organizacional reflejado en la rápida modernización de los sistemas administrativos de sus organizaciones públicas. Además, el proceso de implementación ha sido ágil, el grado de aceptación del sistema por parte de los empleados públicos parece ser alto, y el esfuerzo cuenta con un buen nivel de compromiso de la alta gerencia. Esto se explica en parte por las condiciones estables de gobernabilidad y en parte por los incentivos simbólicos y materiales que motivan a los empleados y gerentes a participar en los acuerdos organizados para cada estrategia de modernización.

Pero a pesar de las fortalezas de los dos esfuerzos, no sabemos aún si en realidad ellos van a promover, como sus gestores aspiran, una gestión 
pública orientada a resultados, con el consecuente mejoramiento sistemático de la gestión pública, de los servicios y bienes que ella produce, y de las políticas públicas bajo las cuales opera. La evidencia parece sugerir que, a pesar de las limitaciones y obstáculos en su implementación, hay razones para ser optimistas. Sin embargo, desde el punto de vista histórico, es tal vez demasiado pronto para emitir juicios al respecto. Lo que si queda claro es que a medida que estos dos esfuerzos siguen desarrollándose en la práctica, sería conveniente contar con un proceso de investigación empírica que permita recoger lecciones útiles para otros países y aumentar nuestros conocimientos sobre la naturaleza de la gestión pública como herramienta de modernización en América Latina.

Efectivamente, este aprendizaje es importante porque la estrategia de cada país para responder a las exigencias generadas a partir de los procesos de reforma del Estado tiene importantes implicaciones para la consolidación de sus estructuras democráticas, y por tanto, afecta la capacidad de gobernar de sus dirigentes. En este sentido, el fracaso o el éxito de los esfuerzos de evaluación de la gestión pública como un componente crítico del proceso de modernización, está directamente relacionado con los problemas de legitimidad y gobernabilidad en los estados latinoamericanos. Si esto es cierto, entonces el diseño e implementación de sistemas de medición y evaluación deben ser concebidos como parte de una amplia estrategia de gestión pública con importantes visos políticos y democráticos. Aspiraciones relacionadas con esta actividad en el contexto público son: la rendición de cuentas (en inglés, accountability); la calidad del servicio; la equidad en su prestación; la productividad, eficiencia y efectividad de las organizaciones; la transparencia de la gestión y el control del gasto público. Vista desde esta perspectiva, la evaluación de la gestión no es solamente un problema técnico o gerencial de interés exclusivo para profesionales de la gestión. La evaluación es además un asunto político que concierne tanto a gobemantes como a gobernados. 
Artículo publicado en la Revista del CLAD Reforma y Democracia, Caracas, oo 19 (Feb. 2001).

1 No hay duda que estos cambios a su vez han sido influenciados por la nueva filosofía de la gestión, pero también es claro que los profundos cambios que experimenta la región tienen también en parte un origen endógeno.

2 La coyuntura se refiere al gobierno del Presidente Samper, quien fue acusado de utilizar dineros del narcotráfico para su campaña y, por tanto, pasó gran parte de su administración en actitud defensiva más que ofensiva.

\section{Bibliografía}

АвітвоL, Pablo. (sin fecha, circa 1994), "La Evaluación como parte integral de la gestión pública en Colombia”. Documento de trabajo de la División Especial de Evaluación, Departamento Nacional de Planeación, Bogotá, Colombia.

Bresser Pereira, Luiz Carlos. (1997), "State Reform in the 1990s: Logic and Control Mechanisms" Presentado en el Congreso Internacional del CLAD, Venezuela, Octubre, 1997.

Bryson, John M. (1988), Strategic Planning for Public and Nonprofit Organizations. A Guide to Strengthening and Sustaining Organizational Achievement, Jossey-Bass Publishers, San Francisco.

Cabrero M., Enrique. (1995), Del Adminitrador al Gerente Público. Instituto Nacional de Administración Pública, México.

Caiden, Gelald E.; CAIDEn, Naomi J. (sin fecha, circa 1997), "Approaches and Guidelines for Monitoring, Measuring and Evaluating Performance in Public Sector Programs", presentado en la Conferencia sobre Modernización del Estado organizada por Development Alternatives, Inc, Washington DC.

CAmpbell, Tim. (1997), Innovations and Risk Taking. The Engine of Reform in Local Government in Latin America and the Caribbean. The International Bank for Reconstruction and Development/The World Bank.

CEPAL. (1998), El Pacto Fiscal: Fortalezas, debilidades, desafíos. Chile: Comisión Económica para América Latina y el Caribe, Naciones Unidas.

CIMGP (Comité Interministerial de Modernización para la Gestión Pública). (1996), Guías para la Planificación Estratégica en los Servicios Públicos. Internet: CIMGPa: http://www.modernizacion.cl/cedoc/guias.indikorgpag2.html CIMGPb: http://www.modernizacion.cl/cedoc/guias.indikolgpag1.html

CLAD. (1998), "Una Nueva Gestión Pública para América Latina”. Centro Latinoamericano de Administración para el Desarrollo, Caracas, Venezuela. 
Dirección de Presupuesto. (1999), Guía Metodológica para la Formulación de Programas de Mejoramiento de La Gestión. PMG Ley 19.553. Santiago de Chile, Comité Técnico, Programa de Mejoramiento de la Gestión, SUBDERE-SEGPRES-DIPRES.

Escovar, Silvia. (1996), "Evaluación de la Gestión Pública en Colombia". En: Hacia una Gestión Pública Orientada a Resultados, DNP, Lito Camargo, Ltda., Sta. Fé de Bogotá, pp. 13-24.

Figueroa, Ramón. (1998), "Evaluación del Desempeño en Chile". En: Gestión para el Cambio. CIMGP, Dolmen Ediciones, Chile, pp. 209-212.

Gandour, Miguel. (1996), "La Evaluación de Resultados en el Contexto de un Proceso de Democratización”. En: Hacia una Gestión Pública Orientada a Resultados. DNP Lito Camargo, Ltda. Sta. Fé de Bogotá, pp. 25-30.

Hogan, Cornelius D. (1999), "Mobilizing Communities: Data Strategies that Contribute to Better Outcomes for People," presented at 21 st Annual Research Conference Association of Public Policy and Management, New York City.

(1999a), Vermont Communities: Using Results to Stregthen Services for Families and Children. Agency of Human Services. Vermont.

Holloway, J., Lewis, J., M allory, G. (1995), Performance Measurement and Evaluation, Sage Publications, London.

KetтL, Donald F. (1997), "The Global Revolution in Public Management: Driving Themes, Missing Links". Journal of Policy Analysis and Management 16: 3, Summer, pp. 446-462. (2000), The Global Public Management Revolution Washington, Brookings Institution Press.

Lynn, Lawrence. (1998), "Requiring Bureaucracies to Perform: What Have we Learned from the US Government Performance and Results Act? " Working Paper Series: 983. The Harris School, University of Chicago.

Ministerio de la Secretaría General de la Presidencia. (1997), Por un Estado al Servicio de la Gente. Programa de Modernización de la Gestión Pública. Chile: Comité Interministerial de Modernización de la Gestión Pública.

Naim, M. (1995), Latin American's Journey to the Market: From Macroeconomic Shocks to Institutional Therapy, California, ICS Press.

Ospina, Sonia. (1999a), "Las Múltiples Dimensiones de la Innovación en el Contexto Latinoamericano". Presentado en el Congreso Internacional del CLAD, México, Octubre, 1999.

. (1999b), “Nueva Gestión Pública en América Latina?”. Presentado en el Taller "Ahora Las Instituciones: Desarrollo Institucional para el Desarrollo Humano en América Latina”. UNDP/IIG, México, 1999.

(1997), Sinergia: Diseño, Organización y Operación de un Modelo de Evaluación para Impulsar la Gestión Pública Orientada a Resultados. Informe de Consultoría para el Departamento Nacional de Planeación. Bogotá, Colombia.

Prats, J. (1997), "La Reforma del Estado y de la Administración Pública”. Presentado en el Congreso Internacional del CLAD, Venezuela, Octubre, 1997.

ReID, Gary. (sin fecha, circa 1997), "La Modernización del Sector Público Orientada hacia el Desempeño en los Países en Desarrollo: Enfrentar el Desafío de la Implementación". Presented at Conference on State Modernization organized by Development Alternatives, Inc, Washington DC.

Rist, Ray C. (1990), Program Evaluation and the Management of Government: Patterns and Prospects across eight Nations, transaction Publishers, New Brunswick.

Rhodes, R.A.W. (ed). (1991), “The New Public Management”. Public Administration. Vol 69: 1.

Sмiтн, Dennis C. (1997), "What Public Managers Learn from Police Reform in New York? COMSTAT and the Promise of Performance Management". Presentado en 19th Annual Research Conference Association of Public Policy and Management, Washington DC. 
Smith, Dennis C., Barnes, Robin A. (1998), "Making Management Count: Toward theorybased Performance Management". Presentado en 20th Annual Research Conference Association for Public Policy and Management, New York.

Waisbluth, Mario. (1998), "Indicadores de Desempeño en la Gestión”. En: Gestión para el Cambio. CIMGP, Dolmen Ediciones, Chile, pp. 213-216.

World Bank. (1997), Colombia Paving the Way for a Results-Oriented Public Sector. World Bank Report No. 15300-CO. Washington DC. 


\section{Resumo \\ Resumen \\ Abstract}

\section{Avaliação da gestão pública: conceitos e aplicações no caso latino-americano Sonia Ospina Bozzi}

Existem atualmente importantes esforços para criar indicadores de gestão em toda América Latina. Sem dúvida, são poucos os casos nos quais se pode falar sobre a utilização sistemática destes indicadores para avaliar a gestão e organizações, programas e políticas públicas. Pressupõe-se a existência de instrumentos de avaliação com capacidade para abordar os níveis micro e macro da gestão, situando-os dentro de um contexto mais amplo que se relaciona com outros níveis da ação estatal, tanto nacional quanto local, e suas interações com outros atores da sociedade civil. A aplicação de uma perspectiva sistêmica de avaliação representa uma agenda urgente dentro do esforço por avançar no processo de modernização do Estado na região. Existem vários países que avançaram na direção adequada, entre eles, destacam-se os casos do Chile, Colômbia e Costa Rica.

Neste trabalho, utilizam-se os casos sul-americanos como ponto de referência para reflexão sobre a natureza da avaliação da gestão pública e explorar em que medida, na América Latina, esta prática representa mais que uma simples técnica administrativa e qual tem sido sua função como ferramenta de modernização do Estado. Este objetivo se desenvolve a partir de uma análise comparativa entre os casos do Chile e Colômbia, considerando três questões para se analisar tanto os elementos conceituais, quanto a efetividade das práticas nos contextos particulares: "para que avaliar?"; "o que avaliar e em que nível fazê-lo?"; e "o que fazer com a informação obtida?". Aos casos colombianos e chilenos, somam-se alguns exemplos de casos nos EUA, dado que neste país houve contribuições práticas e teóricas sobre a avaliação da gestão pública a partir de seu estudo empírico, em contraste com a ausência de pesquisa sistemática sobre os esforços existentes nos casos latino-americanos.

A partir destas três questões e dos casos específicos, este trabalho desenvolve a idéia de que, dada a estreita relação entre gestão e políticas públicas, a avaliação para a gestão pública será mais efetiva como instrumento de modernização na medida que envolva os três níveis da ação social: o macro, o médio e o micro, e na medida que gere capacidade para estabelecer conexões entre estes níveis. Isto garante àqueles que tomam decisões pertinentes ao nível macro utilizar informação dos níveis inferiores e vice-versa, criando assim maior coerência nos diferentes níveis da administração pública. $\mathrm{O}$ trabalho também destaca a necessidade de se organizar uma agenda de pesquisas empíricas para estudar mais profundamente os esforços nacionais de avaliação da gestão pública na região.

\section{Evaluación de la gestión pública: conceptos y aplicaciones en el caso latinoamericano}

\section{Sonia Ospina Bozzi}

Existen en la actualidad importantes esfuerzos para crear indicadores de gestión en toda América Latina. Sin embargo, son pocos los casos en los cuales se puede hablar de la utilización sistemática de estos indicadores para evaluar la gestión de organizaciones, programas y políticas públicas. Ello supone tener instrumentos de evaluación con capacidad para cubrir los niveles micro y meso de la gestión, pero ubicándolos dentro de un contexto más amplio que se relaciona con otros niveles de la acción estatal, tanto nacional como local, y sus interacciones con otros actores de la sociedad civil. La aplicación de una perspectiva sistémica de evaluación representa una agenda urgente dentro del esfuerzo por avanzar en el proceso de modernización del Estado en la región. Existen ya varios países que han avanzado en la dirección adecuada. Entre ellos, se destacan los casos de Chile, Colombia y Costa Rica.
Revista do

Serviço

Público

Ano 52

Número 1

Jan-Mar 2001

Sonia Ospina

Bozzi es

directora del

Programa de

Doctorado y

profesora en la

Escuela de Post

Grado de

Administración

Pública en la

Universidad de

Nueva York. 
En este trabajo se utilizan los dos casos sudamericanos como punto de referencia para reflexionar sobre la naturaleza de la evaluación de la gestión pública y explorar en qué medida en América Latina esta práctica representa más que una simple técnica administrativa y cuál ha sido su función como herramienta de modernización del Estado. Este objetivo se desarrolla alrededor de un análisis comparativo entre los casos de Chile y Colombia, a partir de tres preguntas que presentan dilemas interesantes para analizar, tanto los elementos conceptuales como la efectividad de las prácticas en contextos particulares. Son ellas: ¿para qué evaluar?, ¿qué evaluar y a qué nivel hacerlo? y ¿qué hacer con la información recogida? Además de los casos colombiano y chileno, se incluyen también algunos ejemplos de casos en los Estados Unidos, dado que en este país se han hecho interesantes aportes prácticos y teóricos sobre la evaluación de la gestión pública a partir de su estudio empírico, en contraste con la ausencia de investigación sistemática sobre los esfuerzos existentes en los casos latinoamericanos.

A partir de estas tres preguntas y de los casos específicos, el trabajo desarrolla la idea de que, dada la estrecha relación entre gestión y políticas públicas, la evaluación para la gestión pública será más efectiva como instrumento de modernización en la medida que abarque los tres niveles de la acción social, el macro, meso y micro, y en la medida que genere capacidad para establecer conexiones entre estos niveles. Esto garantiza que quienes toman decisiones pertinentes al nivel macro de la acción estatal puedan utilizar información de los niveles inferiores, y viceversa, creándose así una mayor coherencia en los distintos niveles de la administración pública. El trabajo, además, señala la urgencia de organizar una agenda de investigación empírica para estudiar más a fondo los esfuerzos nacionales de evaluación de la gestión pública en la región.

\section{Evaluation of public management: concepts and applications in the Latin American case}

\section{Sonia Ospina Bozzi}

Currently, there are major efforts at setting up management indicators throughout Latin America. However, few cases show the systematic use of such indicators to evaluate the management of organisations, programmes and public policies. This entails the use of evaluation tools covering micro and meso management to a wider context as related to other State levels, both national and local, and their interactions with other players within civil society. The application of a systemic evaluation is an urgent part of the agenda, in the effort to advance State modernisation process in the region. Several countries have already progressed in the appropriate direction, they include Chile, Colombia and Costa Rica.

This paper uses two South-American cases to discuss the evaluative nature of public management and to explore whether this practice in Latin America, is merely an administrative technique and to discuss its role in State modernisation. A comparative analysis of Chile and Colombia is used, using three questions which show important dilemmas to be analysed, both in terms of their conceptual components and their effectiveness in a given context: "what is evaluation for?", "what should be evaluated and at which level?" and "what should be done with the data collected?" In addition to the Colombian and Chilean cases, some examples from the United States are included. The United States has made significant practical and theoretical contributions to the evaluation of public management based on its empirical study, as opposed to the lack of systematic research into existing efforts in Latin American cases.

From these questions and specific cases, this paper develops the concept that, given the close relationship between management and public policies, evaluation for public management will be more effective as a modernisation instrument as long as it covers the three levels of social action - macro, meso and micro - , and as long as it develops the ability to make connections among such levels. This ensures that decision makers at the macro level in State action use information from lower levels and vice versa, resulting in a greater coherence within the different levels of public administration. This paper also states the need to organise an empirical research agenda to delve into each country's efforts at evaluating public management in the region. 